\title{
Apolipoprotein M promotes the anti-inflammatory effect of high- density lipoprotein by binding to scavenger receptor $\mathrm{BI}$
}

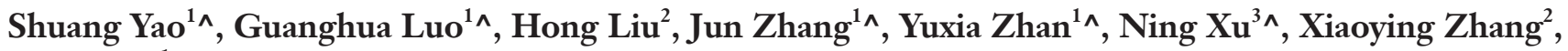 \\ Lu Zheng ${ }^{1 \wedge}$
}

${ }^{1}$ Clinical Medical Research Center, the Third Affiliated Hospital of Soochow University, Changzhou, China; ${ }^{2}$ Department of Cardiothoracic Surgery, the Third Affiliated Hospital of Soochow University, Changzhou, China; ${ }^{3}$ Division of Clinical Chemistry and Pharmacology, Department of Laboratory Medicine, Lunds University, Lunds, Sweden

Contributions: (I) Conception and design: S Yao, L Zheng; (II) Administrative support: G Luo; (III) Provision of study materials or patients: H Liu; (IV) Collection and assembly of data: S Yao, J Zhang; (V) Data analysis and interpretation: All authors; (VI) Manuscript writing: All authors; (VII) Final approval of manuscript: All authors.

Correspondence to: Lu Zheng. Clinical Medical Research Center, the Third Affiliated Hospital of Soochow University, 185 Juqian Street, Changzhou 213003, China. Email: qz.zhenglu@163.com.

Background: Inflammation participates pivotally in the pathogenesis of atherosclerosis. Apolipoprotein $M($ apoM) is a high-density lipoprotein (HDL)-associated plasma protein that affects HDL metabolism and shows various anti-inflammatory functions in atherosclerosis. In this study, we aim to determine whether apoM is expressed in peripheral blood mononuclear cells (PBMCs) and promoted the anti-inflammatory effect of HDL by combing with scavenger receptor BI (SR-BI).

Methods: The expression of apoM in PBMCs is detected by a confocal fluorescence microscope and flow cytometry. The interactions between apoM and SR-BI are detected with co-immunoprecipitation. The multiplexed Luminex xMAP assay detects the inflammatory factors induced by apoM $\mathrm{HDL}^{+} \mathrm{Hnd}_{\text {apoM }}^{-}$ HDL in inflammatory cell model.

Results: ApoM is expressed on $\mathrm{CD}_{14}{ }^{+}$monocytes, $\mathrm{CD}^{+} \mathrm{T}$ cells, and CD19 ${ }^{+} \mathrm{B}$ cells, CD16 $6^{+}$and $\mathrm{CD}^{+} 6^{+}$ NK cells. CD $14^{+}$monocytes have the highest ratio of apoM ${ }^{+}$cells. ApoM ${ }^{+}$HDL, apoM ${ }^{-}$HDL, and recombinant apoM protein could be co-precipitated with SR-BI on the surface of human THP-1 monocytic leukemia cells. In vitro, apoM $\mathrm{M}^{+} \mathrm{HDL}$ induces significantly less expression of tumor necrosis factor- $\alpha$ (TNF- $\alpha$ ), interleukin-6 (IL-6) and IL-1 $\beta$ than apoM- $M^{-}$DL.

Conclusions: ApoM was expressed on all PBMCs. ApoM interacted with SR-BI on THP-1. ApoM ${ }^{+}$HDL has a more significant anti-inflammatory effect than apoM ${ }^{-}$HDL.

Keywords: Apolipoprotein M (apoM); high-density lipoprotein (HDL); inflammatory effect; peripheral blood mononuclear cell (PBMC); scavenger receptor class B type I

Submitted Aug 06, 2020. Accepted for publication Nov 26, 2020.

doi: $10.21037 /$ atm-20-7008

View this article at: http://dx.doi.org/10.21037/atm-20-7008

^ ORCID: Shuang Yao: 0000-0003-3686-5404; Guanghua Luo: 0000-0001-8339-2828; Jun Zhang: 0000-0002-1826-6099; Yuxia Zhan: 0000-0002-5823-6695, Ning Xu: 0000-0002-6064-329X; Lu Zheng: 0000-0002-5563-4348. 


\section{Introduction}

Atherosclerosis (AS) is an inflammatory reaction to a vascular injury, which begins with an arterial wall injury and endothelial dysfunction leading to inflammation and the release of inflammatory factors. Currently, it has been well established that central steps in atherogenesis include development of: (I) endothelial dysfunction and increased expression of adhesion molecules and increased permeability; (II) macrophages being recruited to the lesion site by adhering to activated endothelial cells and entering into the subendothelial cell space; (III) deposition and oxidation of low-density lipoprotein (LDL) in the arterial intima and macrophages take up lipid deposit particles and ultimately transform into foam cells, which can form a necrotic core, a typical feature of the instability of advanced plaques, leading to the rupture of plaques and further acute life-threatening (1).

Aside from reverse cholesterol transport (RCT), the next most-recognized function of HDL is in antiinflammatory regulation. Recently, the anti-inflammatory properties of HDL were found in several types of cells, including endothelial cells and monocytes. For example, apolipoprotein A-I (apoA-I), the major HDL protein, might attenuate nuclear factor- $\kappa \mathrm{B}(\mathrm{NF}-\kappa \mathrm{B})$ activation by reducing toll-like receptor-4 recruitment into lipid rafts (2). Human recombinant HDL inhibited the early pro-inflammatory changes and suppressed the endothelial expression of vascular cell adhesion molecule-1, intercellular adhesion molecule-1, and monocyte chemoattractant protein-1 (MCP-1) in rabbits (3). SR-BI is a multi-ligand membrane protein receptor that binds to apoA-I on HDL, promoting the selective uptake and reverse transport of cholesterol. Studies in mice and humans have demonstrated that a functional defect of SR-BI can cause atherosclerotic lesions and cardiovascular diseases, such as myocardial infarction and stroke (4). Tao et al. demonstrated that macrophage SR-BI could led to the activation of the downstream molecules phosphoinositide 3-kinase (PI3K) and Rasrelated C3 botulinum toxin substrate 1 (Rac1), phagocytosis and elimination of apoptotic cells in atherosclerotic lesions, thereby reducing necrosis, inhibiting inflammatory responses and exerting an anti-atherosclerotic effect (5). It is also found to mediate HDL-induced anti-inflammatory effects in macrophages via the inhibition of $\mathrm{NF}-\kappa \mathrm{B}$ activation (6). The interaction between apoA-I and SR$\mathrm{BI}$ inhibits NF- $\mathrm{KB}$ translocation and decreases the plasma inflammatory mediator levels via the PI3K/Akt pathway (7). However, because of the variety of SR-BI ligands, it is unclear whether SR-BI interacts with other apolipoproteins on HDL. The exact molecular mechanism behind the antiinflammatory effects of HDL is still unknown.

ApoM, an apolipoprotein that is primarily present in HDL, is involved in lipid and lipoprotein metabolism $(8,9)$. ApoM was found to play an essential role in the formation of pre- $\beta-H D L$ and cholesterol efflux to HDL (10). In plasma, apoM is transported with HDL, and apoM $\mathrm{M}^{+} \mathrm{HDL}$ accounts for $5 \%$ of the total HDL. Recently, the antiinflammatory functions of apoM, mediated by HDL, have attracted increasing attention (11-14). The ApoM gene is on chromosome 6 and located close to inflammatory genes, including $T N F-\alpha$ and $T N F-\beta$ (8). Feingold $e t$ al. showed that lipopolysaccharides (LPS) decreased apoM expression (15). According to recent reports, apoM also protected LPS-treated mice from death and organ injury (16). Christoffersen et al. found that apoM $\mathrm{M}^{+} \mathrm{HDL}$ particles were more effective in cholesterol efflux, compared with apoM- $M^{-}$DL particles (10). Previously, we found the RCT was disrupted in the $S R-B I\left(S R-B I^{--}\right)$gene knockout mice, which then increased total cholesterol levels and apoM expression levels in serum (17). Therefore, we hypothesized that apoM might also be involved in SR-BImediated cholesterol transport. An excessive accumulation of cholesterol in macrophages was regarded as a trigger for the inflammatory process. Our research group found that apoM-induced inhibition of inflammatory responses may occur via the S1PR1 pathway in apoM knockout mice and overexpressed human hybrid endothelial cell line (18). These findings suggested that apoM might function as an anti-inflammatory factor.

Our earlier studies showed that apoM is expressed primarily in the liver and the proximal renal tubules. Small amounts could also be observed in fetal liver and kidney tissues (19). The biological efficacy of apoM expression in PBMCs is still unknown. Macrophages are the essential inflammatory effector cells in atheromatous plaque formation, and it is vital to understand the apoM expression profiling in PBMCs, for investigating the effect of apoM in inflammatory processes. In this study, we took macrophages as the research object and focused on the anti-inflammatory effects of apoM in the peripheral circulation.

At the beginning of this study, we found apoM was expressed on PBMCs by using confocal microscopy and flow cytometry. Afterward, we purified the apoM $M^{+} \mathrm{HDL}$ and apo $\mathrm{M}^{-} \mathrm{HDL}$ by using affinity chromatography to determine whether apoM is the dominant element in the binding of HDL and SR-BI. Then, we analyzed the differences in the 
Table 1 The sources of the primary and secondary antibodies

\begin{tabular}{lcc}
\hline Cells or molecule & Primary antibody (source) & Secondary antibody (source) \\
\hline ApoM & Rabbit anti-human apoM (GeneTex) & PE-goat anti-rabbit (Proteintech) \\
T cell & FITC-mouse anti-human CD3 (BD) & - \\
Monocyte & FITC-mouse anti-human CD14 (BD) & - \\
NK cell & PE-Cy5-mouse anti-human CD56 (BD) & - \\
NK cell & APC-mouse anti-human CD16 (BioLegend) & - \\
B cell & PerCP-mouse anti-human CD19 (BioLegend) & - \\
Isotype control & Rabbit IgG (Abcam) & - \\
\hline
\end{tabular}

ApoM, apolipoprotein M; NK cell, natural killer cell; CD, cluster of differentiation; FITC, fluorescein isothiocyanate; PE-Cy5, phycoerythrinCy5 conjugate; APC, allophycocyanin; PerCP, peridinin-chlorophyll-proteincomplex; PE, R-Phycoerythrin.

secretion of inflammatory factors induced by apoM- $\mathrm{MDL}^{-}$ and apoM $\mathrm{M}^{+} \mathrm{HDL}$ in macrophages. The research novelty lies in the first elucidation of apoM expression in PBMCs; protein co-immunoprecipitation assay provides a more intuitive way to identify the combination between apoM and SR-BI expressed on macrophages. In addition, apoM in plasma mainly exists in HDL particles. We compared the difference of anti-inflammatory effects between apo $\mathrm{M}^{+} \mathrm{HDL}$ and apoM $\mathrm{M}^{-} \mathrm{HDL}$, which is closer to the real situation in vivo.

We present the following article in accordance with the MDAR reporting checklist (available at http://dx.doi. org/10.21037/atm-20-7008).

\section{Methods}

Flow-cytometric analysis and confocal imaging of apoM in PBMCs

The expression of apoM in PBMCs was detected by using a confocal fluorescence microscope (Leica SP8, Heidelberg, Germany). Flow cytometry was used to quantify the percentage of apoM positive cells in subpopulations of PBMCs (BD, Accuri, C6, CA, USA). PBMCs, isolated from the whole blood of a male healthy donor through a FicollHypaque density gradient centrifugation, are classified into various subgroups by using the corresponding antibodies labeled with fluorescein. PBMC cells were fixed with $4 \%$ paraformaldehyde and incubated with a blocking buffer, which comprised phosphate buffer saline (PBS) containing $1 \%$ BSA (Sigma, St. Louis, USA). Samples were randomized into 15 groups and incubated with primary and secondary antibodies in dark conditions for $30 \mathrm{~min}$. Before the flow cytometry, we constructed cell smears from each group for observation under confocal microscopy. The sources of the primary and secondary antibodies are summarized in Table 1 . Table 2 lists all groupings and corresponding conjugated fluorochromes.

All procedures performed in this study involving human participants were in accordance with the Declaration of Helsinki (as revised in 2013). The Ethics Committee approved the protocol of this study of the Third Affiliated Hospital of Soochow University [2011(ke)No. 162]. Informed consent has been taken from the donor.

\section{Purification of apoM ${ }^{+} \mathrm{HDL}$ and apoM $\mathrm{HDL}^{-}$}

A mouse anti-human apoM monoclonal antibody is coupled to a HiTrap NHS-activated HP column (GE, USA) by covalent binding. Two components of the HDL protein $\left(\right.$ apoM ${ }^{+}$HDL and apoM ${ }^{-}$HDL particles) were separated by using an affinity chromatography method. We conjugated the column with an anti-apoM antibody (Abgent Biotech, China), loaded it twice with HDL (ProSpec-Tany, TechnoGene, Israel) diluted in PBS, and collected the effluent. After loading was completed, we used $100 \mathrm{mM}$ glycine ( $\mathrm{pH}$ 2.2) to elute the column and collect the eluent. A BCA protein quantitation assay (BestBio, China) was used to determine the concentration of the separated proteins.

\section{Cell culture and immunoprecipitation}

Human THP-1 monocytic leukemia cells (Cat.No SCSP$567)$ were obtained from the cell culture center of the Chinese Academy of Medical Sciences and cultured in RPMI1640 medium supplemented with 10\% FCS and 
Table 2 Groupings and corresponding conjugated fluorochromes

\begin{tabular}{ll}
\hline Grouping & Added antibody \\
\hline 1 & Cell only \\
2 & FITC-CD3 \\
3 & PerCP-CD19 \\
4 & FITC-CD14 \\
5 & PE-Cy5-CD56 \\
6 & APC-CD16 \\
7 & ApoM-Ab and PE-goat anti-rabbit \\
8 & ApoM-Ab, PE-goat anti-rabbit, and FITC-CD3 \\
9 & ApoM-Ab, PE-goat anti-rabbit, and PerCP-CD19 \\
10 & ApoM-Ab, PE-goat anti-rabbit, and FITC-CD14 \\
11 & ApoM-Ab, PE-goat anti-rabbit, and PE-Cy5-CD56 \\
12 & ApoM-Ab, PE-goat anti-rabbit, and APC-CD16 \\
13 & ApoM-Ab, PE-goat anti-rabbit, and FITC-CD3 and \\
14 & PerCP-CD19 \\
15 & ApoM-Ab, PE-goat anti-rabbit, FITC-CD3, APC-CD16, \\
& Isotype control and PE-goat anti-rabbit
\end{tabular}

ApoM, apolipoprotein M; NK cell, natural killer cell; CD, cluster of differentiation; FITC, fluorescein isothiocyanate; PE-Cy5, phycoerythrin-Cy5 conjugate; APC, allophycocyanin; PerCP, peridinin-chlorophyll-proteincomplex; PE, R-Phycoerythrin.

$2 \mathrm{mmol} / \mathrm{L}$ L-glutamine (Gibco, Grand Island, NY, USA). Cells were maintained in a humidified atmosphere with $5 \%$ $\mathrm{CO}_{2}$ at $37^{\circ} \mathrm{C}$. THP-1 cells were incubated with $160 \mathrm{nM}$ PMA (Gene Operation, China) and $100 \mu \mathrm{g} / \mathrm{mL}$ ox-LDL (Peking Union-Biology Co. Ltd., China) to differentiate them into macrophages, as described previously (20). THP-1 cells were then treated for 6 hours with either apoM $M^{-}$HDL $(50 \mu \mathrm{g} / \mathrm{mL})$ or apoM $M^{+}$HDL $(50 \mu \mathrm{g} / \mathrm{mL})$ and recombinant human apoM protein $(1 \mu \mathrm{g} / \mathrm{mL})$, respectively. Cells were collected, and total proteins were extracted. Aliquots of cell lysate were incubated with $2 \mu \mathrm{g}$ mouse anti-SR-BI antibody overnight at $4{ }^{\circ} \mathrm{C}$. The immune complex was captured with Protein A/G PLUS-Agarose (sc-2003, Santa Cruz, CA, USA) by incubation for 0.52 hours. Beads were washed with added sample buffers to remove any non-bound proteins from the precipitated complex. The beads were boiled in reducing the SDSPAGE sample loading buffer, and SDS-PAGE analyzed the covered eluate.

\section{Identification of the different inflammatory factors secreted by macrophages following incubation with apoM- $H D L$ or apo $M^{+} H D L$}

THP- 1 cells $\left(5 \times 10^{6} /\right.$ well $)$ were differentiated into foam cells using $160 \mathrm{nM}$ of PMA and $100 \mu \mathrm{g} / \mathrm{mL}$ of ox-LDL for 24 hours, respectively. Induced THP-1 cells were divided into three groups (each group had six replicates): group 1 , with a culture medium containing $20 \mu \mathrm{g} / \mathrm{mL}$ apo $\mathrm{M}^{-}$HDL; group 2, with a culture medium containing $20 \mu \mathrm{g} / \mathrm{mL}$ apoM $\mathrm{M}^{+} \mathrm{HDL}$; and group 3, a control group. After induction for 24 hours in the culture media above, the cell supernatants were collected, and the expressions of TNF- $\alpha$, IL-10, IL-6, IL-1 $\beta$, and MCP-1 were detected by a Luminex 200 assay (Milliplex Map Kit, Human Magnetic Bead, MAPmate ${ }^{\text {TM }}$ Buffer Kit, Millipore, Billerica, MA, USA). Each experiment was replicated three times.

\section{Statistical analysis}

Multiple comparisons were performed with one-way analysis of variance (ANOVA), followed by Dunnett's multiple comparison test. $\mathrm{P}<0.05$ was considered to indicate a statistically significant difference. The statistical analysis was conducted using Graph-Pad Prism Software version 5.0 (San Diego, CA, USA). Values are expressed as mean \pm SEM.

\section{Results}

\section{The expression of apoM in PBMCs}

Inflammation-induced by immune cells is the key factor in all the stages of atherogenesis, from lesion formation to plaque rupture. The first step was to confirm the apoM expression on immune cells to investigate whether apoM takes part in this process. Confocal images showed that each subpopulation of PBMCs, including the $\mathrm{CD} 19^{+} \mathrm{B}$ cell (Figure 1A), the $\mathrm{CD}^{+} \mathrm{T}$ cell (Figure $1 B$ ), the $\mathrm{CD} 16^{+} \mathrm{CD} 56^{+}$, the $\mathrm{CD} 16^{-} \mathrm{D} 56^{+} \mathrm{NK}$ cells (Figure $1 B$ ), as well as the $\mathrm{CD} 14^{+}$ monocyte (Figure 1C) expressed apoM. The flow cytometric analysis showed that the expression ratio of apoM on $\mathrm{CD} 14^{+}$ monocytes was $10 \%$, which was the highest ratio among the five groups. The other expression ratios of apoM on $\mathrm{CD}^{+} \mathrm{T}$ cells, CD19 $9^{+}$cells, $\mathrm{CD} 56^{+} \mathrm{NK}$ cells, and $\mathrm{CD} 16^{+} \mathrm{NK}$ cells were $5.6 \%, 8.3 \%, 2.4 \%$ and $4.1 \%$, respectively (Figure 2, Table 3). 

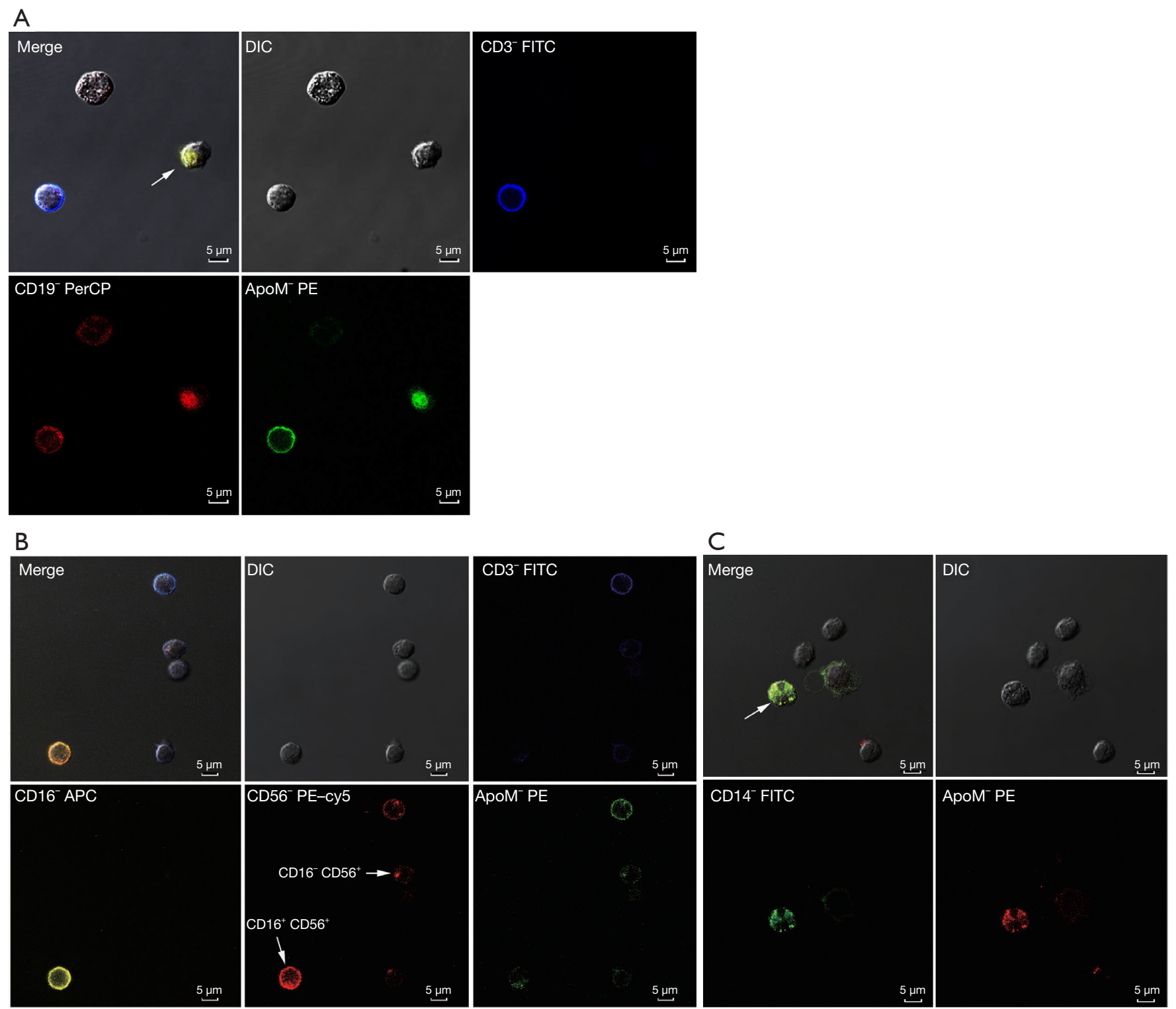

Figure 1 Confocal fluorescence microscopic images of apo $\mathrm{M}^{+}$cells in PBMCs. Localization was carried out by incubation of cells with rabbit anti-human apoM or rabbit IgG (isotype control). (A) ApoM is expressed on CD19 ${ }^{+}$B cells (marked with an arrow). Blue: $\mathrm{CD} 3^{+}$; red: $\mathrm{CD}^{+} 9^{+}$; green: apoM+. (B) ApoM is expressed on $\mathrm{CD}^{+} \mathrm{T}$ cells and the CD16 ${ }^{+} \mathrm{CD} 56^{+}$, the CD16 ${ }^{-}$5 $6^{+} \mathrm{NK}$ cells (marked with arrows). Blue: $\mathrm{CD}^{+}$; yellow: $\mathrm{CD} 16^{+}$; red: $\mathrm{CD} 56^{+}$, green: apoM+. (C) ApoM is expressed on CD14+ monocyte (marked with an arrow). Green: CD14+; red: apoM $\mathrm{M}^{+}$. ApoM, apolipoprotein M; PBMCs, peripheral blood mononuclear cells. Merge, a overlapped image from multiple fluorescence channels. DIC, differential-interference-contrast image.

\section{Isolated apoM $M^{+} H D L$ bound to SR-BI}

We put together a mouse anti-human apoM monoclonal antibody to a HiTrap NHS-activated HP column by the covalent binding mode. The two components in the HDL protein $\left(\right.$ apoM $M^{+} \mathrm{HDL}$ and apoM- $\left.\mathrm{HDL}\right)$ were separated according to the HiTrap NHS-activated HP column purification protocol. The dot blot test analyzed the effluent and elution liquid and Western blot, both with 

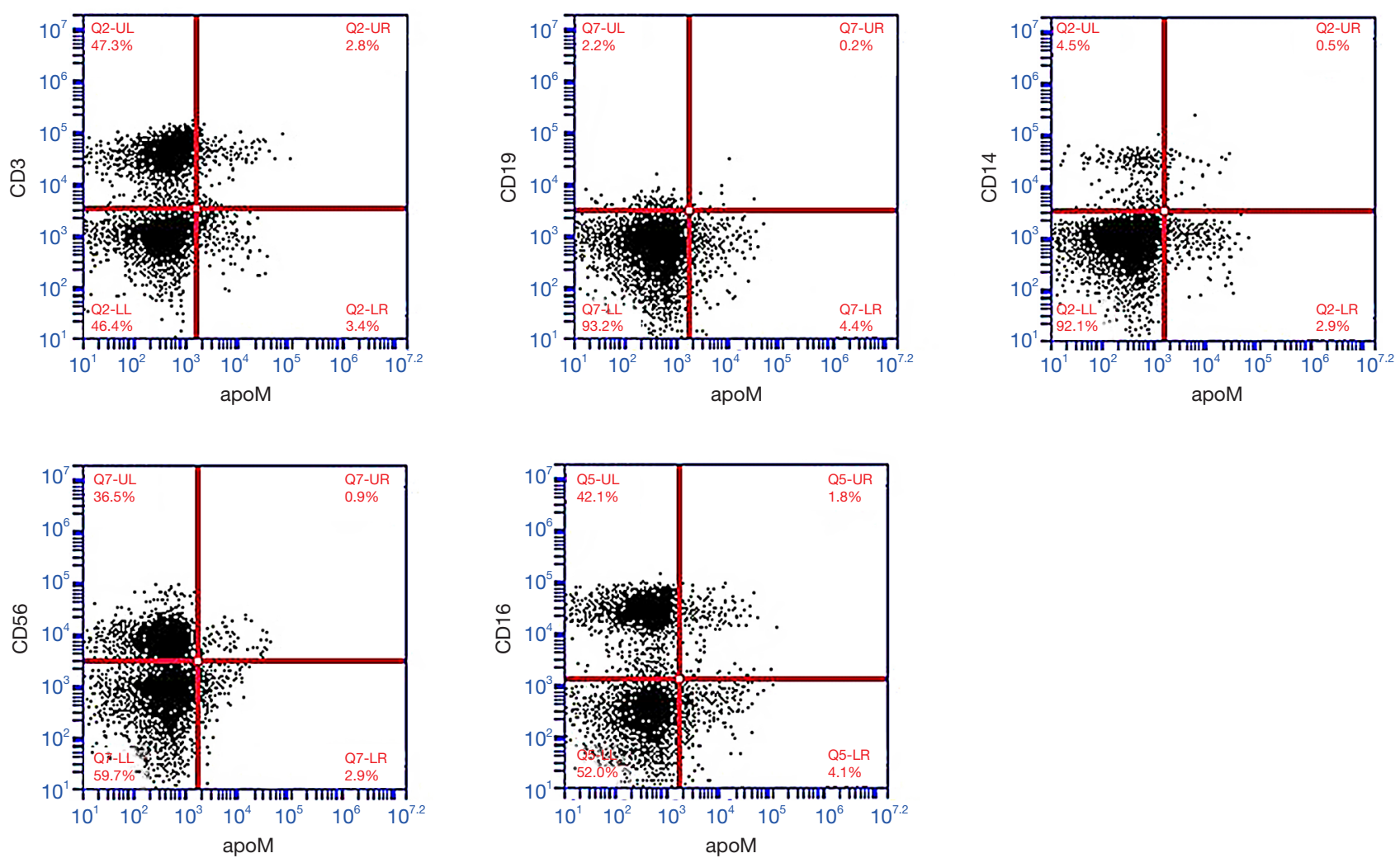

Figure 2 Flow cytometry analysis of the expression ratios of apoM ${ }^{+}$cells in PBMCs. T cell, B cell, monocytes and NK cell popolations are identified using CD3, CD19, CD14, CD56 and CD16 (Y-axis), respectively. Upper right quadrant shows CD and apoM double positive cells. The percentage of each quadrant means the ratio of cells in this quadrant relative to all PBMCs. ApoM, apolipoprotein M; PBMCs, peripheral blood mononuclear cells.

Table 3 The ratios of cells with apoM ${ }^{+}$to total PBMCs and target subpopulations

\begin{tabular}{lcc}
\hline \multirow{2}{*}{ Cell marker } & \multicolumn{2}{c}{ ApoM $^{+}$} \\
\cline { 2 - 3 } & Relative to PBMCs & Relative to subpopulations \\
\hline CD3 $^{+}$ & $2.8 \%$ & $5.6 \%$ \\
CD19 $^{+}$ & $0.25 \%$ & $8.3 \%$ \\
CD14 $^{+}$ & $0.5 \%$ & $10 \%$ \\
CD56 $^{+}$ & $0.9 \%$ & $2.4 \%$ \\
CD16 $^{+}$ & $1.8 \%$ & $4.1 \%$ \\
\hline
\end{tabular}

Relative to PBMCs: apoM ${ }^{+}$cell $\& \mathrm{CD}^{+}$cell/all PBMCs. Relative to subpopulations: apoM ${ }^{+}$cell/ $/ \mathrm{CD}^{+}$cell. apoM, apolipoprotein M; PBMCs, peripheral blood mononuclear cells; CD, cluster of differentiation. using the apoM antibody. The results proved that the apoM protein could be detected in the unpurified HDL protein and the elution liquid, but not in the effluent liquid (Figure $3 A, B)$. The final concentration of the purified $\operatorname{apoM}^{-}$HDL and apoM $M^{+}$HDL particles were 3.23 and $0.34 \mathrm{mg} / \mathrm{mL}$, respectively.

Second, the purified apoM $M^{-}$HDL and apoM $M^{+}$HDL particles, together with recombinant human apoM, were used to treat induced THP-1 cells to determine the combination of apoM and SR-BI. The results of the coimmunoprecipitation showed that the recombinant human apoM, human apoM- $\mathrm{HDL}$, and human apoM $\mathrm{M}^{+} \mathrm{HDL}$ particles could interact with SR-BI on the surfaces of the THP-1 cells (Figure 3C,D). 


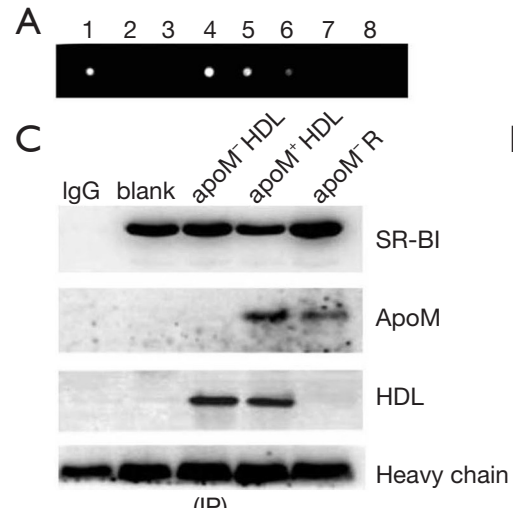

(IP)

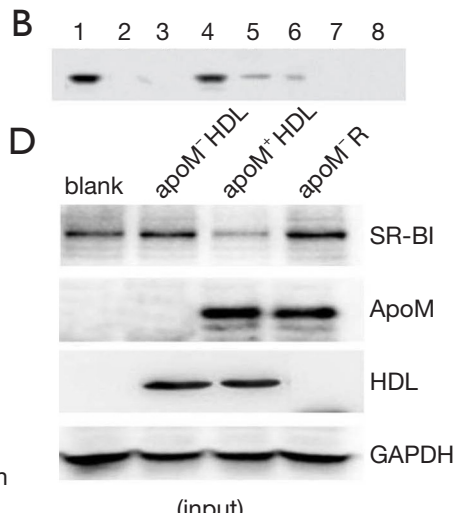

(input)

Figure 3 Identification of the interactions between SR-BI and apoM by co-immunoprecipitation. (A,B) Dot blot and Western blot analysis of apoM in effluent liquid and elution fractions. 1: unpurified HDL (diluted with PBS 1:10); 2: effluent liquid (the flow-through after apoM ${ }^{+}$ HDL binding to the column, diluted with PBS 1:10); 3: wash liquid (the flow-through after washing with the wash buffer); 4-8: elution

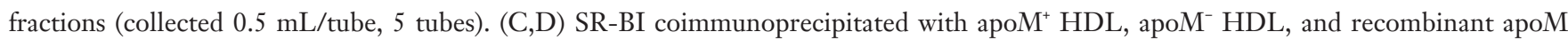
protein in THP-1 cells. THP-1 cells were incubated with $160 \mathrm{nM}$ PMA and $100 \mu \mathrm{g} / \mathrm{mL}$ ox-LDL for 24 hours, respectively. Fifty $\mu \mathrm{g} / \mathrm{mL}$ of both apoM $\mathrm{M}^{+} \mathrm{HDL}$ and apoM $\mathrm{M}^{-} \mathrm{HDL}$, as well as $1 \mu \mathrm{g} / \mathrm{mL}$ of recombinant apoM protein, was added to the medium for 6 hours before cell harvest. Cell lysates were immunoprecipitated using SR-BI antibodies coated with protein A beads. Immunoprecipitates were resolved by denaturing SDS-PAGE, then blotted and probed with HDL and apoM antibodies. apoM, apolipoprotein M; SR-BI, scavenger receptor BI; HDL, high-density lipoprotein; PBS, phosphate buffer saline.

\section{Differential effects of apoM- $H D L$ and apo $M^{+} H D L$ in stimulating the secretion of inflammatory factors in macrophages}

Macrophage activation triggers cytokine release and the recruitment of monocytes, which are past events in the development of atherosclerosis. Therefore, we investigated macrophages to study the anti-inflammatory effects of apoM. THP-1 macrophages were differentiated from monocytes with a PMA treatment for 24 hours. Ox-LDL was used to set up a macrophage-derived foam cell model. The results showed that both apoM $\mathrm{M}^{-} \mathrm{HDL}$ and apo $\mathrm{M}^{+}$ HDL could down-regulate the secretion of TNF- $\alpha$, IL6 , IL-1 $\beta$, and MCP-1 in THP-1 cells, but apoM ${ }^{+}$HDLinduced decreases of TNF- $\alpha$, IL- 6 , and IL- $1 \beta$ were more significant $(\mathrm{P}=0.0411,0.00379$, and 0.0101 , respectively) than in the apo $\mathrm{M}^{-} \mathrm{HDL}$ treatment group. The expression of IL-10, induced by both apoM $\mathrm{M}^{-} \mathrm{HDL}$ and apoM $\mathrm{M}^{+} \mathrm{HDL}$, was increased, compared with the control group $(\mathrm{P}=0.0096$ and 0.0043 ) (Figure 4). IL-10 is a cytokine with antiinflammatory properties, according to earlier research. Interestingly, MCP-1 showed no marked differences in inhibition between apoM $\mathrm{M}^{+} \mathrm{HDL}$ and $\mathrm{apoM}^{-} \mathrm{HDL}$.

\section{Discussion}

HDL has been proposed to exert several pleiotropic effects. Along with its leading roles in RCT, HDL possesses anti-inflammation, anti-oxidation, anti-thrombosis, and endothelial integrity preservation properties, all of which might be because of proteins and lipids riding on the HDL (21). ApoM belongs to the lipocalin superfamily, all of which exhibit distinct properties in lipid binding, lipid transport, and immune function (22). To explore whether apoM had anti-inflammatory effects, we first detected the expression of apoM in the PBMCs of healthy donors. Results showed that apoM was expressed on CD $14^{+}$ monocytes, $\mathrm{CD}^{+} \mathrm{T}$ cells, and $\mathrm{CD} 19^{+} \mathrm{B}$ cells, and $\mathrm{CD} 16^{+}$ and $\mathrm{CD} 56^{+} \mathrm{NK}$ cells. However, the ratios of the apoM ${ }^{+}$ cells in each subgroup are different. We assumed that this is because of the various differentiation stages that those immune cells were in at the time.

Interestingly, $\mathrm{CD} 14^{+}$cells have the highest ratio of apo $\mathrm{M}^{+}$cells, compared with the other monocytes. CD14 is expressed mainly on macrophages, the central immune cells in the atherosclerotic process. Macrophages engulf lipid material and eventually convert them to foam cells, which 

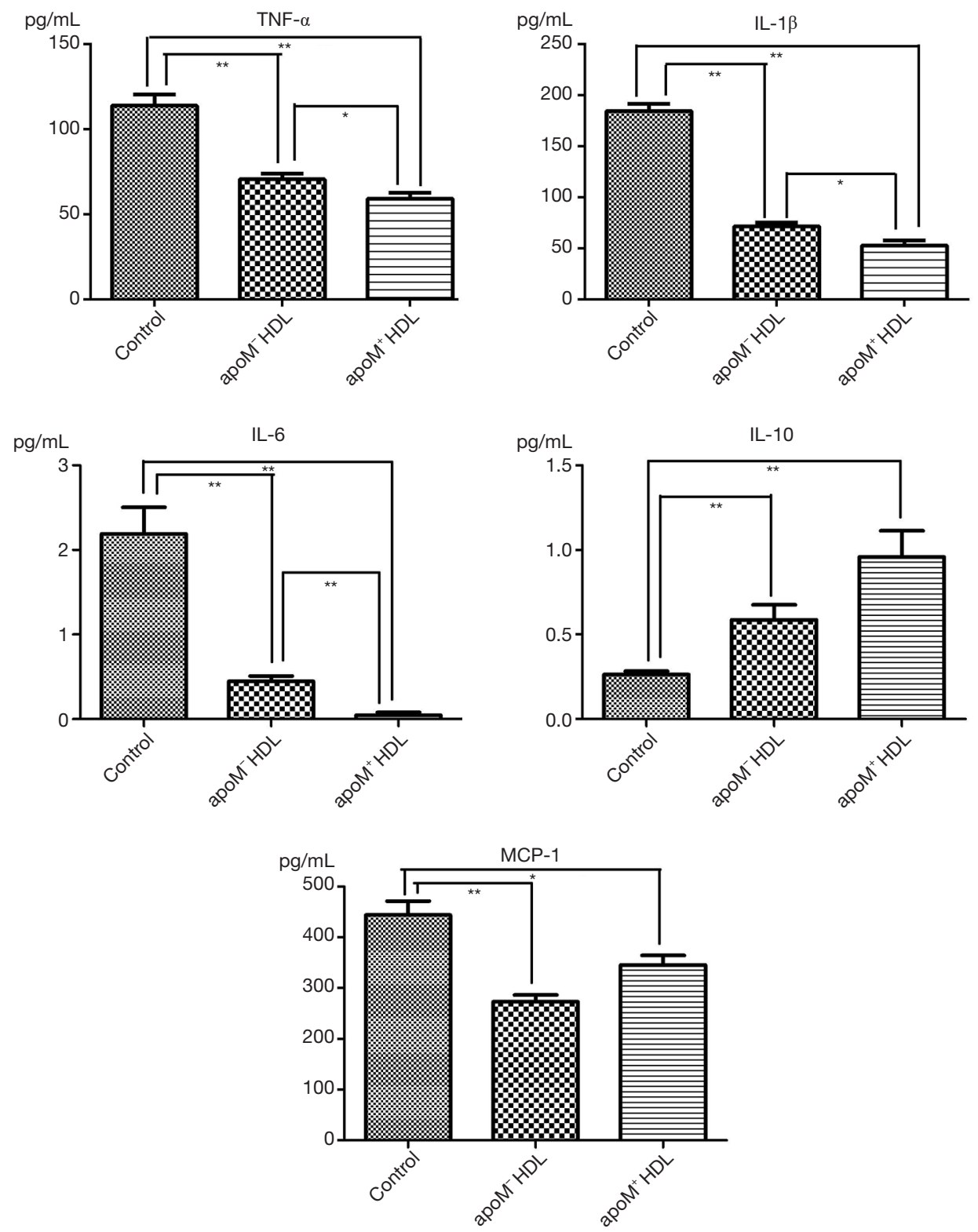

Figure 4 Evaluation of inflammatory cytokine secretions in macrophages stimulated with apoM ${ }^{-} \mathrm{HDL}_{\text {or }}$ apoM ${ }^{+} \mathrm{HDL}$ THP-1 cells were differentiated into macrophages by incubation with $160 \mathrm{nM}$ PMA and $100 \mu \mathrm{g} / \mathrm{mL}$ ox-LDL for 24 hours, respectively. Following differentiation, $20 \mu \mathrm{g} / \mathrm{mL}$ of both apoM $\mathrm{M}^{+} \mathrm{HDL}$ or apoM $\mathrm{M}^{-} \mathrm{HDL}$ is added to the medium. After a 24 hours incubation, the secretion of inflammatory cytokines, including TNF- $\alpha$, IL-10, IL-6, IL-1 $\beta$, and MCP-1, in the supernatants were detected by Luminex 200 (*, P<0.05; **, $\mathrm{P}<0.01)$. ApoM, apolipoprotein M; HDL, high-density lipoprotein; TNF- $\alpha$, tumor necrosis factor- $\alpha$; IL, interleukin; MCP-1, monocyte chemoattractant protein-1.

compose the structure of atheromatous plaques. That apoM had the highest expression ratio in macrophages suggested apoM might take part in the pathological lesions of AS. Changes in the expression levels of apoM, from monocytes to macrophages, and its anti-inflammatory properties should be further ascertained in the future.

Atherosclerosis is a vascular inflammatory response process that occurs after an injury. Mononuclear cells exudate from the arterial intima and differentiate into macrophages. These macrophages then take up lipids, 
including ox-LDL, by receptors on their surface and form foam cells, which then assemble to form fatty streaks. During the progression of atherosclerosis, macrophages also release chemotactic factors and inflammatory factors, including TNF- $\alpha$, IL-6, IL-1 $\beta$, and MCP-1 (23). These cytokines can be broadly classified as proinflammatory and proatherogenic (such as IL-1, IL-6, and TNF- $\alpha$ ) or as antiinflammatory and antiatherogenic (such as IL-10 and IL1RA) (24). TNF- $\alpha$ activates endothelial cells to express adhesion molecules as well as proinflammatory cytokine and chemokine receptors, and support recruitment of activated leukocytes to an inflammatory lesion (25). IL$1 \beta$ regulate activation of macrophages and differentiation of Th17 cells. IL-6 is an upstream inflammatory cytokine that plays a central role in propagating the downstream inflammatory response responsible for atherosclerosis. IL10 is antiatherogenic and suppresses activation of Th1 cells and macrophages, contributing to survival of B cells and antibody production (26). Blaho et al. found apoMbound S1P inhibited lymphopoiesis (27). These findings suggest that apoM has some immunological functions. To gain a better understanding of the function of apoM, we isolated apo $\mathrm{M}^{+} \mathrm{HDL}$ and apoM $\mathrm{M}^{-} \mathrm{HDL}$ proteins by affinity chromatography using prepacked columns and compared the anti-inflammatory activity between two subfractions. In this study, we found that apoM $M^{+} \mathrm{HDL}$ and apoM- $\mathrm{HDL}$ could downregulate the expression of IL-6, IL-1 $\beta$, and MCP-1 in inflammatory macrophages.

Further, analyses showed that the downregulated expression of TNF- $\alpha$, IL- 6 , and IL- $1 \beta$ was more significant in the apoM $\mathrm{M}^{+} \mathrm{HDL}$ group than in the apoM $\mathrm{M}^{-}$HDL group. There was no significant difference in the expression of MCP-1 between the two groups. MCP-1 regulates monocyte recruitment and the activation of T-lymphocyte subpopulations in atherosclerotic cardiovascular disease. We considered that the inhibition of the MCP-1 pathway might not be related to apoM, but might include other apolipoproteins, including apoA-I. We also found that apo $M^{+}$HDL significantly upregulated the expression of IL-10, compared with the control group. IL-10, an anti-inflammatory cytokine, plays a crucial role in lipid deposition and inflammation in atherosclerotic lesions and is confirmed to be a protective factor in atherosclerosis. All these results suggest apoM $\mathrm{M}^{+} \mathrm{HDL}$ might have a more substantial anti-inflammatory effect than apoM $\mathrm{M}^{-} \mathrm{HDL}$ in vitro. However, does apoM have anti-inflammatory activity in vivo? In future study, we will compare the antiinflammatory effects of apoM $\mathrm{MDL}^{+}$and apoM- $\mathrm{HDL}$ in a mouse model of inflammation. And further analysis whether the anti-inflammatory effect of apoM could prevent the progression of atherosclerosis.

Many studies have detailed the various signaling pathways generated by the interaction of HDL with cell surface receptors like SR-BI. According to the recent study, HDL isolated from SR-BI ${ }^{-/}$mice also exhibited a decreased capability of suppressing inflammatory cytokines in macrophages (28). SR-BI has been shown to modulate the susceptibility to LPS-induced tissue injury (29) and the ability of S1P (sphingosine 1 phosphate) to interact with its receptor, linking SR-BI to regulating inflammation (30). ApoM was the reported physiological carrier of S1P on HDL. Therefore, we suspected that apoM could promote the anti-inflammatory effects of HDL by binding to SRBI. The binding of purified apoM $\mathrm{M}^{+} \mathrm{HDL}$ and apoM $\mathrm{M}^{-} \mathrm{HDL}$ proteins to SR-BI, which was expressed on the surface of THP-1 cells, was detected by co-immunoprecipitation. Our results showed that all apo $M^{+} \mathrm{HDL}$, apo $\mathrm{M}^{-} \mathrm{HDL}$, and recombinant apoM protein could be co-precipitated with SR-BI. A combination of apoM ${ }^{-}$HDL and SR-BI could be ascribed to apoA-I. ApoM also interacted with SRBI on THP-1. However, added studies will be needed to determine whether the combination influences the SR-BI downstream signaling pathway. In order to confirm whether the combination of apoM and SR-BI has physiological function, in the following experiments, we will use SR-BI antagonists to block the binding site and investigate whether SR-BI contributes to the anti-inflammatory function of apoM.

However, cellular cholesterol levels modulate macrophage inflammatory responses. The increased cellular cholesterol content resulting from reduced cellular cholesterol efflux contributes to the hyperinflammatory responses in these cells in response to stimuli for ATPbinding cassette transporter deficient macrophages (31). The evidence from our research in mouse peritoneal macrophages showing apo $M^{+}$HDL had a more substantial effect on promoting cholesterol efflux than apoM- $\mathrm{M}^{-} \mathrm{HDL}(\mathrm{P}<0.05$, data will be published in another article). Therefore, we hypothesize apoM modulates the macrophage inflammatory responses partly by affecting the content and distribution of cholesterol in cells.

Our data altogether proved that apoM $\mathrm{M}^{+} \mathrm{HDL}$ has a more substantial anti-inflammatory effect than apoM ${ }^{-}$HDL. Since apoM could interact with SR-BI on THP-1, we assumed that this might be the reason that apoM enhanced the anti-inflammatory effect of HDL, but subsequent 
experiments are needed to prove this effect. Given the fact that macrophage inflammation is a critical feature in the development of atherosclerosis, our findings supply evidence that apoM may exert a protective effect against atherosclerotic lesion formation through modulating macrophage inflammatory responses.

\section{Acknowledgments}

Funding: This work was supported by the National Natural Science Foundation of China (Nos. 81201352, 81370372), the Jiangsu Provincial Natural Science Foundation of China (Nos. BK2012154, BK20130244, BK20151179), the International Cooperation Item of Changzhou (No. CZ20150012), and the Changzhou High-Level Medical Talents Training Project (No. 2016ZCLJ002).

\section{Footnote}

Reporting Checklist: The authors have completed the MDAR reporting checklist. Available at http://dx.doi.org/10.21037/ atm-20-7008

Data Sharing Statement: Available at http://dx.doi. org/10.21037/atm-20-7008

Conflicts of Interest: All authors have completed the ICMJE uniform disclosure form (available at http://dx.doi. org/10.21037/atm-20-7008). The authors have no conflicts of interest to declare.

Ethical Statement: The authors are accountable for all aspects of the work in ensuring that questions related to the accuracy or integrity of any part of the work are appropriately investigated and resolved. All procedures performed in this study involving human participants were in accordance with the Declaration of Helsinki (as revised in 2013). The Ethics Committee approved the protocol of this study of the Third Affiliated Hospital of Soochow University [2011(ke)No. 162]. Informed consent has been taken from the donor.

Open Access Statement: This is an Open Access article distributed in accordance with the Creative Commons Attribution-NonCommercial-NoDerivs 4.0 International License (CC BY-NC-ND 4.0), which permits the noncommercial replication and distribution of the article with the strict proviso that no changes or edits are made and the original work is properly cited (including links to both the formal publication through the relevant DOI and the license). See: https://creativecommons.org/licenses/by-nc-nd/4.0/.

\section{References}

1. Xu H, Jiang J, Chen W, et al. Vascular Macrophages in Atherosclerosis. J Immunol Res. 2019;2019:4354786.

2. Cheng AM, Handa P, Tateya S, et al. Apolipoprotein A-I attenuates palmitate-mediated NF-kappaB activation by reducing Toll-like receptor-4 recruitment into lipid rafts. PLoS One 2012;7:e33917.

3. Yin K, Agrawal DK. High-density lipoprotein: a novel target for antirestenosis therapy. Clin Transl Sci 2014;7:500-11.

4. Ma B, Jia J, Wang X, et al. Differential roles of Scavenger receptor class B type I: A protective molecule and a facilitator of atherosclerosis. Mol Med Rep 2020;22:2599-604.

5. Tao H, Yancey PG, Babaev VR, et al. Macrophage SRBI mediates efferocytosis via Src/PI3K/Rac1 signaling and reduces atherosclerotic lesion necrosis. J Lipid Res 2015;56:1449-60.

6. Kimura T, Tomura H, Mogi C, et al. Role of scavenger receptor class $\mathrm{B}$ type I and sphingosine 1-phosphate receptors in high density lipoprotein-induced inhibition of adhesion molecule expression in endothelial cells. J Biol Chem 2006;281:37457-67.

7. Ren K, Lu YJ, Mo ZC, et al. ApoA-I/SR-BI modulates S1P/S1PR2-mediated inflammation through the PI3K/ Akt signaling pathway in HUVECs. J Physiol Biochem 2017;73:287-96.

8. Xu N, Dahlback B. A novel human apolipoprotein (apoM). J Biol Chem 1999;274:31286-90.

9. Zheng L, Feng Y, Shi Y, et al. Intralipid decreases apolipoprotein $M$ levels and insulin sensitivity in rats. PLoS One 2014;9:e105681.

10. Christoffersen C, Jauhiainen M, Moser M, et al. Effect of apolipoprotein $\mathrm{M}$ on high density lipoprotein metabolism and atherosclerosis in low density lipoprotein receptor knock-out mice. J Biol Chem 2008;283:1839-47.

11. Huang XS, Zhao SP, Hu M, et al. Apolipoprotein M likely extends its anti-atherogenesis via anti-inflammation. Med Hypotheses 2007;69:136-40.

12. Li H, Liu Y, Wang L, et al. High apolipoprotein M serum levels correlate with chronic obstructive pulmonary disease. Lipids Health Dis 2016;15:59.

13. Poti F, Simoni M, Nofer JR. Atheroprotective role of 
high-density lipoprotein (HDL)-associated sphingosine-1phosphate (S1P). Cardiovasc Res 2014;103:395-404.

14. Shen T, Wu WM, Du WH, et al. Positive association between serum apolipoprotein $M$ levels and hepatitis B virus DNA load in HBeAg-negative chronic hepatitis B. Lipids Health Dis 2016;15:210.

15. Feingold KR, Shigenaga JK, Chui LG, et al. Infection and inflammation decrease apolipoprotein $\mathrm{M}$ expression. Atherosclerosis 2008;199:19-26.

16. Kurano M, Tsuneyama K, Morimoto Y, et al. Apolipoprotein M Protects Lipopolysaccharide-Treated Mice from Death and Organ Injury. Thromb Haemost 2018;118:1021-35.

17. Feng YH, Zheng L, Wei J, et al. Increased apolipoprotein $\mathrm{M}$ induced by lack of scavenger receptor $\mathrm{BI}$ is not activated via HDL-mediated cholesterol uptake in hepatocytes. Lipids Health Dis 2018;17:200.

18. Wang M, Luo GH, Liu H, et al. Apolipoprotein $M$ induces inhibition of inflammatory responses via the S1PR1 and DHCR24 pathways. Mol Med Rep 2019;19:1272-83.

19. Zhang XY, Jiao GQ, Hurtig M, et al. Expression pattern of apolipoprotein $M$ during mouse and human embryogenesis. Acta Histochem 2004;106:123-8.

20. Kurosaka K, Watanabe N, Kobayashi Y. Production of proinflammatory cytokines by phorbol myristate acetatetreated THP-1 cells and monocyte-derived macrophages after phagocytosis of apoptotic CTLL-2 cells. J Immunol 1998;161:6245-9.

21. Santos-Gallego CG, Badimon JJ, Rosenson RS. Beginning to understand high-density lipoproteins. Endocrinol Metab Clin North Am 2014;43:913-47.

22. Luo G, Zhang X, Nilsson-Ehle P, et al. Apolipoprotein M. Lipids Health Dis 2004;3:21.

Cite this article as: Yao S, Luo G, Liu H, Zhang J, Zhan Y, $\mathrm{Xu} \mathrm{N}$, Zhang $\mathrm{X}$, Zheng L. Apolipoprotein $M$ promotes the anti-inflammatory effect of high-density lipoprotein by binding to scavenger receptor BI. Ann Transl Med 2020;8(24):1676. doi: 10.21037/atm-20-7008
23. Moore KJ, Sheedy FJ, Fisher EA. Macrophages in atherosclerosis: a dynamic balance. Nat Rev Immunol 2013;13:709-21.

24. Ridker PM. Anticytokine Agents: Targeting Interleukin Signaling Pathways for the Treatment of Atherothrombosis. Circ Res 2019;124:437-50.

25. McKellar GE, McCarey DW, Sattar N, et al. Role for TNF in atherosclerosis? Lessons from autoimmune disease. Nat Rev Cardiol 2009;6:410-7.

26. Fatkhullina AR, Peshkova IO, Koltsova EK. The Role of Cytokines in the Development of Atherosclerosis. Biochemistry (Mosc) 2016;81:1358-70.

27. Blaho VA, Galvani S, Engelbrecht E, et al. HDL-bound sphingosine-1-phosphate restrains lymphopoiesis and neuroinflammation. Nature 2015;523:342-6.

28. Cao J, Xu Y, Li F, et al. Protein markers of dysfunctional HDL in scavenger receptor class B type I deficient mice. J Transl Med 2018;16:155.

29. Song GJ, Kim SM, Park KH, et al. SR-BI mediates high density lipoprotein (HDL)-induced anti-inflammatory effect in macrophages. Biochem Biophys Res Commun 2015;457:112-8.

30. Lee MH, Appleton KM, El-Shewy HM, et al. S1P in HDL promotes interaction between SR-BI and S1PR1 and activates S1PR1-mediated biological functions: calcium flux and S1PR1 internalization. J Lipid Res 2017;58:325-38.

31. Zhu X, Lee JY, Timmins JM, et al. Increased cellular free cholesterol in macrophage-specific Abca1 knock-out mice enhances pro-inflammatory response of macrophages. J Biol Chem 2008;283:22930-41.

(English Language Editor: J. Chapnick) 